\title{
Interior layer structure in the Newtonian blown film
}

\author{
J. J. Shepherd* J. C. Bennett ${ }^{\dagger}$ \\ (Received 29 October 2004, revised 27 July 2005)
}

\begin{abstract}
The techniques and methodology of singular perturbation theory analyse the film blowing of an incompressible Newtonian film. An appropriate, physically relevant small parameter guides the method of matched asymptotic expansions to obtain straightforward closed form approximate expression for the film profile throughout the blown region. This then determines such related quantities as the film thickness variation, and the film speed. The results of applying this closed form expression are compared with numerical calculations using the package Maple. The two methods show encouraging consistency.
\end{abstract}

*School of Mathematical and Geospatial Sciences, Royal Melbourne Institute of Technology, Melbourne, Australia. mailto: jshep@rmit.edu.au

${ }^{\dagger}$ School of Mathematical and Geospatial Sciences, Royal Melbourne Institute of Technology, Melbourne, Australia. mailto:s2111773@student.rmit.edu.au

See http://anziamj.austms.org.au/V46/CTAC2004/Shep for this article, (C) Austral. Mathematical Soc. 2005. Published September 1, 2005. ISSN 1446-8735 


\section{Contents}

1 Introduction

C840

2 Governing equations

C842

3 Numerical results

C844

4 Perturbation analysis

C846

5 Results and discussion

C851

References

C853

\section{Introduction}

We present a mathematical analysis of the simplest model of the film blowing process, the widely used industrial process employed to manufacture the thin polymer film widely used in commercial and domestic applications. In reality, any full analysis of the most realistic models of film blowing would involve highly nonlinear problems, reflecting the complex physical and chemical changes occurring during the manufacturing process. However, the relatively unsophisticated model we analyse here avoids many of the mathematical difficulties associated with more realistic models, at the same time retaining a lot of the characteristic solution structure observed.

Prior to considering the mathematical details of this simple model, we describe the essential features of the industrial manufacturing process itself. These are displayed schematically in Figure 1. A thin annular die of radius $R_{0}$, width $E_{0}$ extrudes a tube of molten polymer film at a constant velocity $U_{0}$. Application of an internal pressure difference $\Delta P$ causes this thin film tube to expand to an increased radius, as shown. At the same 


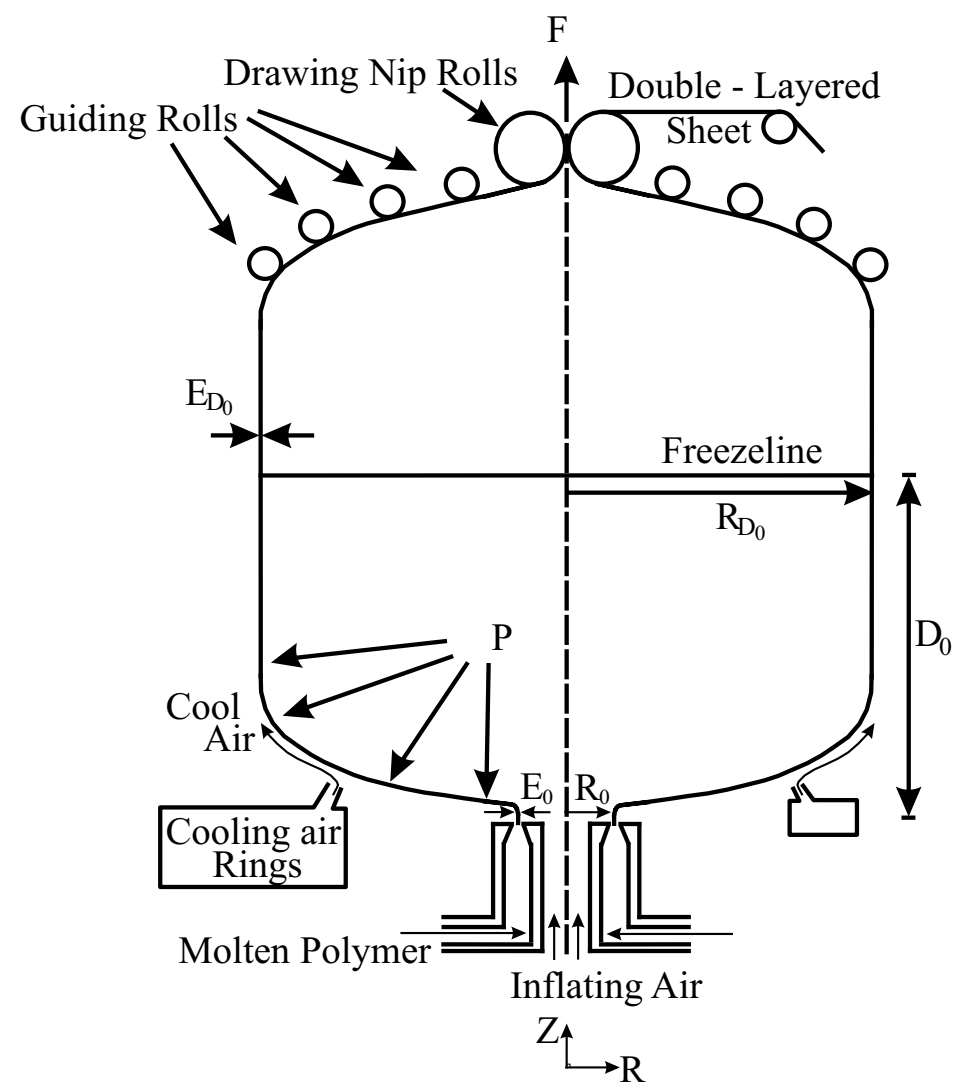

Figure 1: Schematic of film blowing process.

time, this tube or bubble of polymer is cooled by external air jets from an air ring located above the die, causing the film to solidify, eventually reaching a constant radius $R_{D_{0}}$ with thickness $E_{D_{0}}$ at the freezeline, where its speed is $U_{D_{0}}$. Subsequently, the overall bubble shape remains unaltered; with the tube of thin film being rolled flat as a double layered film and drawn off on to an overhead roller.

For a physically realistic range of values of the significant parameters of the system, this film bubble radius expansion occurs over a relatively small 
part of the overall bubble region. Thus, we view this localized expansion region as an interior layer region; appropriate asymptotic methods based on a suitably chosen small parameter yield approximations to the radius profile and other related quantities.

We make the assumption that the material comprising the bubble is an incompressible Newtonian fluid, of constant viscosity $\eta_{0}$. Further, we ignore the effects of gravity on the film, so that it is viewed as a thin shell under tension only from the drawing rolls and the imposed internal pressure $\Delta P$. Moreover, we assume that the bubble is in equilibrium, so that the blowing process is steady, while temperature variations are negligible; that is, the process proceeds isothermally.

While these assumptions are severe simplifications of the real world film blowing process, they lead to a mathematical model displaying the overall features of more sophisticated models.

\section{Governing equations}

The equations describing the film blowing process for materials displaying a range of complexity are well represented in the literature [4]. For the present investigation, we consider only the relatively simple situation of the steady, isothermal, gravity free dilation of an incompressible Newtonian sheet. Under the assumption of axial symmetry, the only relevant spatial variables become the radial distance $R$, measured transverse to the bubble axis; and axial distance $Z$ measured along that axis from the polymer extrusion die. Consequently, the state of the film is described completely by the bubble radius $R(Z)$, its meridional speed $U(Z)$, the film thickness $E(Z)$ and the tension $F(Z)$; all depending only on the axial variable $Z$, with $Z$ ranging from $Z=0$ at the injection point (that is, at the die) to $Z=D_{0}$ at the freezeline. 
Introducing the dimensionless variables

$$
\begin{aligned}
& z=\frac{Z}{D_{0}}, \quad r=\frac{R}{R_{0}}, \quad u(z)=\frac{U(Z)}{U_{0}}, \\
& e(z)=\frac{2 \pi R_{0} U_{0} E(Z)}{Q}, \quad f(z)=\frac{F(Z) R_{0}}{\eta_{0} Q},
\end{aligned}
$$

where $Q$ is the volume flow rate of fluid in the film, $\eta_{0}$ is (as above) its viscosity, whereas $R_{0}, E_{0}, U_{0}$ and $\Delta P$ are as described above, allows us to express the momentum and mass conservation equations as the nonlinear differential equation for $r(z)$

$$
\begin{aligned}
& 2 C^{2} r^{2}\left(f_{0}+B\left(r^{2}-1\right)\right) r^{\prime \prime}-6 C r^{\prime}+4 B r^{3}\left(1+C^{2}\left(r^{\prime}\right)^{2}\right) \\
& -r\left(f_{0}+B\left(r^{2}-1\right)\right)\left(1+C^{2}\left(r^{\prime}\right)^{2}\right)=0
\end{aligned}
$$

where the dimensionless quantities

$$
B=\frac{\pi \Delta P R_{0}^{3}}{\eta_{0} Q}, \quad C=\frac{R_{0}}{D_{0}}, \quad f_{0}=\frac{F_{0} R_{0}}{\eta_{0} Q},
$$

respectively, and $F_{0}=F(0)$, together with the differential equation for $u(z)$,

$$
2 C\left(2 r u^{\prime}+u r^{\prime}\right)-r u\left(f_{0}+B\left(r^{2}-1\right)\right)\left(1+C^{2}\left(r^{\prime}\right)^{2}\right)=0 ;
$$

the algebraic equation for $e(z)$,

$$
r(z) u(z) e(z)=1 ;
$$

and the force condition for

$$
f(z)=f_{0}+B\left(r^{2}-1\right) .
$$

Appropriate boundary conditions at the ends of the bubble are

$$
r(0)=1, \quad r^{\prime}(1)=0, \quad u(0)=1, \quad e(0)=1 .
$$




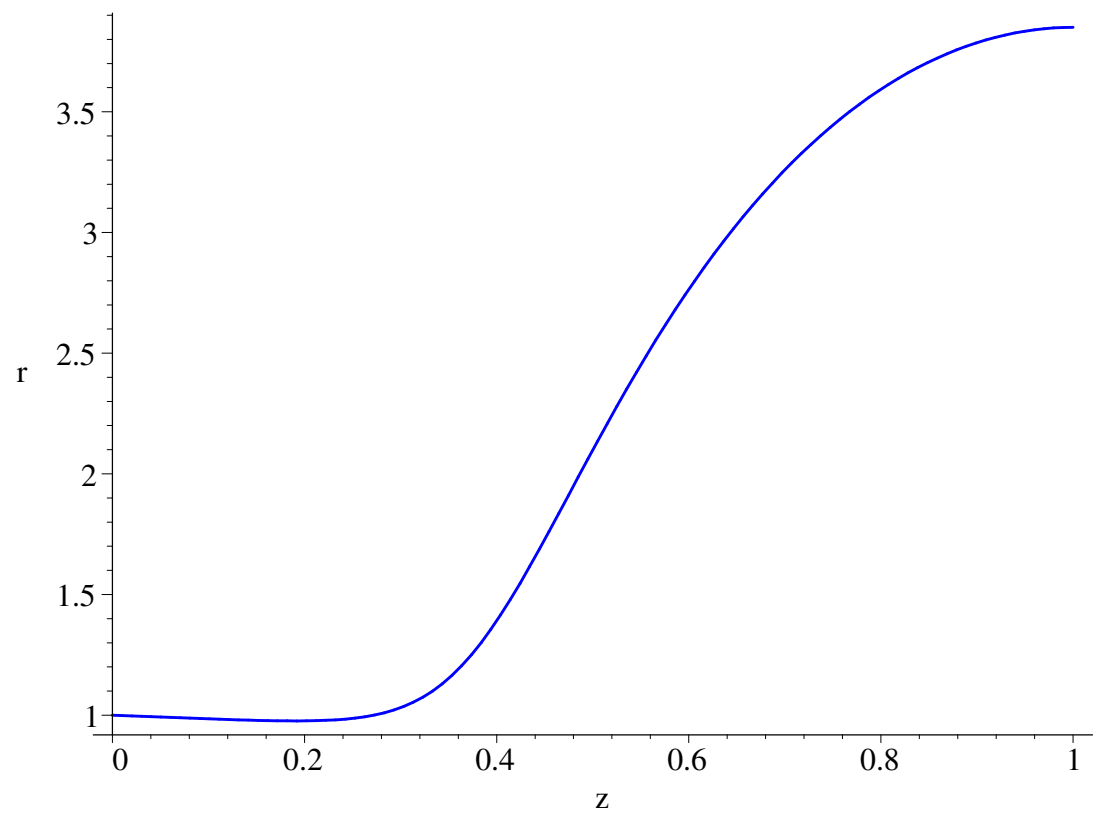

FiguRE 2: Typical bubble radius profile $r(z)$.

Note that Equation (2), while highly nonlinear, is a single equation for $r(z)$ that may, in principle, be solved subject to the boundary conditions (7).

This solution may then be applied to (4) and (7), to obtain expressions for the film speed $u(z)$, while (5), (6) and (7) yield the film thickness $e(z)$ and tension $f(z)$.

\section{Numerical results}

The nonlinear boundary value problem comprising the differential equation (2) together with the boundary conditions (7) cannot be solved analytically; numerical methods must be employed. Typically, we expect a 


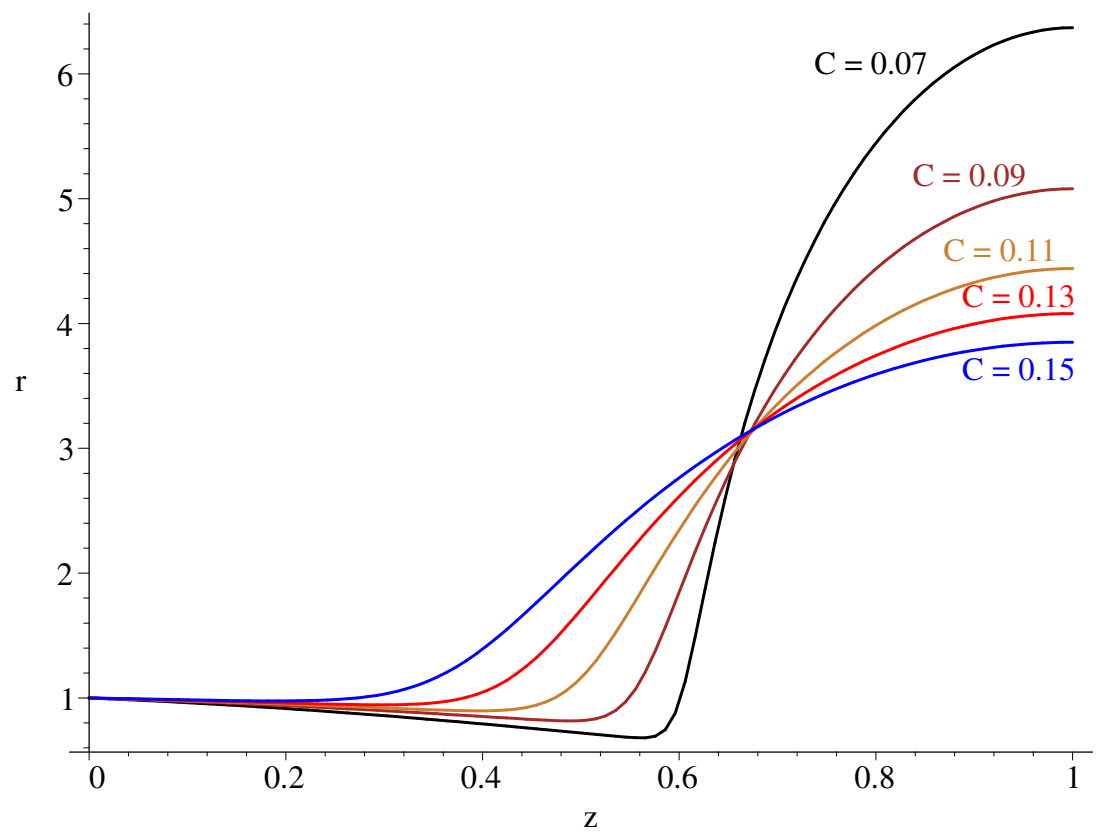

Figure 3: Radius profile $r(z)$ for decreasing $C$-values. Smaller $C$-values correspond to steeper slopes in the transition region.

shooting method to be appropriate, using initial conditions $r(0)=1$ and $r^{\prime}(0)=k$, with $k$ being adjusted to meet the second boundary condition at $z=1[5,6,7]$. However, in the present case, we experienced instability due to the zero slope condition at $z=0[1,2]$. Thus, here a backward shooting technique is employed, by which (2) was integrated from $z=1$ back to $z=0$, with initial conditions

$$
r(1)=\rho_{\mathrm{BU}} \quad \text { and } \quad r^{\prime}(1)=0, \quad \rho_{\mathrm{BU}}=\frac{R_{D_{0}}}{R_{0}},
$$

where $\rho_{\mathrm{BU}}$ is the blow up ratio. A typical bubble radius profile is shown in Figure 2. The radius changes relatively slowly for some distance from the die $(z=0)$; then follows a region of rapid expansion, followed by a third region of again relatively slow variation, until the freezeline $(z=1)$ is reached. Here, 
we are interested in discovering how this region of rapid change ('interior layer') is derived from (2), which of the physical parameters $B, C, f_{0}$ define its structure; and how we may obtain useful approximations to the radius profile. Figure 3 displays the radius profile for fixed values of $B$ and $f_{0}$, and decreasing values of $C-C=0.15,0.13,0.11,0.09$ and 0.07 . The interior layer structure appears to arise as a result of the limiting process $C \rightarrow 0$. Thus, it seems that a perturbation analysis based on this limit may yield useful results. Accordingly, we employ perturbation techniques to construct an approximation to the film bubble profile $r(z)$.

\section{Perturbation analysis}

Rearrange (2) to the form

$$
C^{2} r^{\prime \prime}+\alpha\left(r, r^{\prime}, C\right) r^{\prime}+\beta(r)=0,
$$

where

$$
\alpha\left(r, r^{\prime}, C\right)=\frac{C^{2}\left(3 B r^{2}-f_{0}+B\right) r r^{\prime}-6 C}{2 r^{2}\left(f_{0}+B\left(r^{2}-1\right)\right)},
$$

and

$$
\beta(r)=\frac{\left(3 B r^{2}-f_{0}+B\right) r}{2 r^{2}\left(f_{0}+B\left(r^{2}-1\right)\right)} .
$$

We assume that the interior layer (region of rapid bubble radius variation) is centred at $z=a$, and that $r(a)=\lambda$, where $a$ and $\lambda$ are to be determined. For the numerical results of Section 3 , we expect $a \approx 0.5$ and $\lambda \approx 1.7$. Then view the solution of $(9)$ on $[0,1]$ as the union of solutions $r_{1}(z)$ and $r_{2}(z)$ on $[0, a]$ and $[a, 1]$, with boundary conditions

$$
r_{1}(0)=1, \quad r_{1}(a)=\lambda \quad \text { and } \quad r_{2}(a)=\lambda, \quad r_{2}^{\prime}(1)=0,
$$

respectively, together with suitable smoothness conditions at $z=a$. For $C \rightarrow 0$, the structure of these solutions depends heavily on the (unknown) 


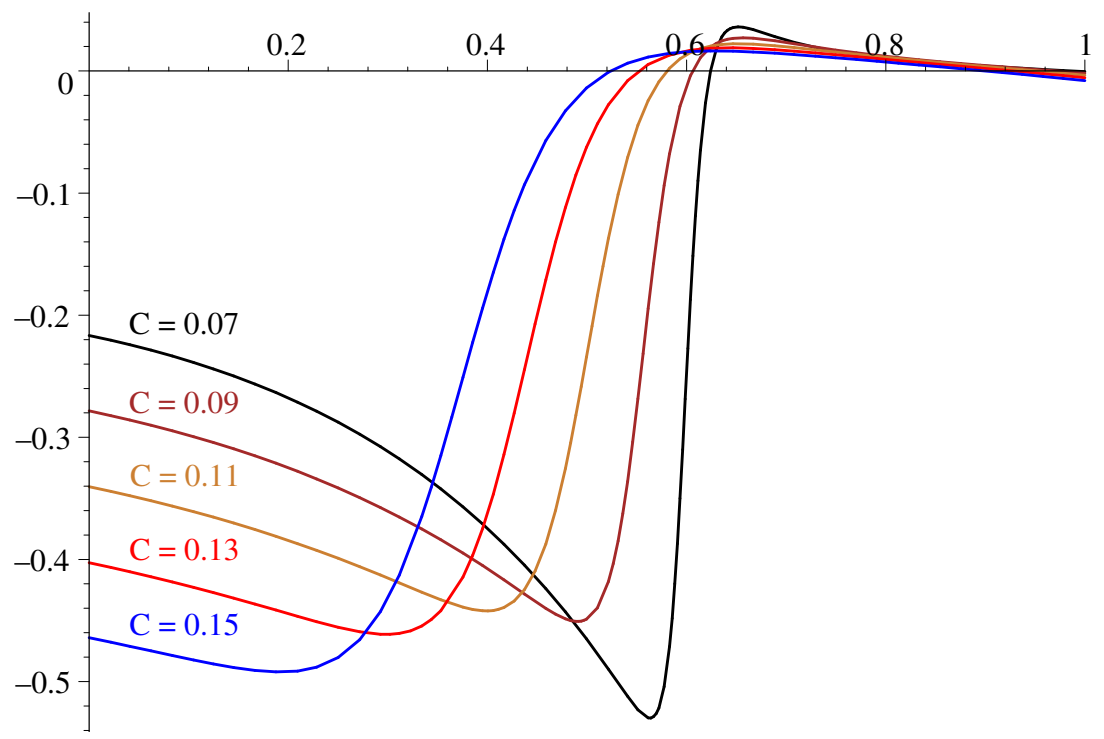

Figure 4: Variation of $\alpha\left(r, r^{\prime}, C\right)$ of Equation (9).

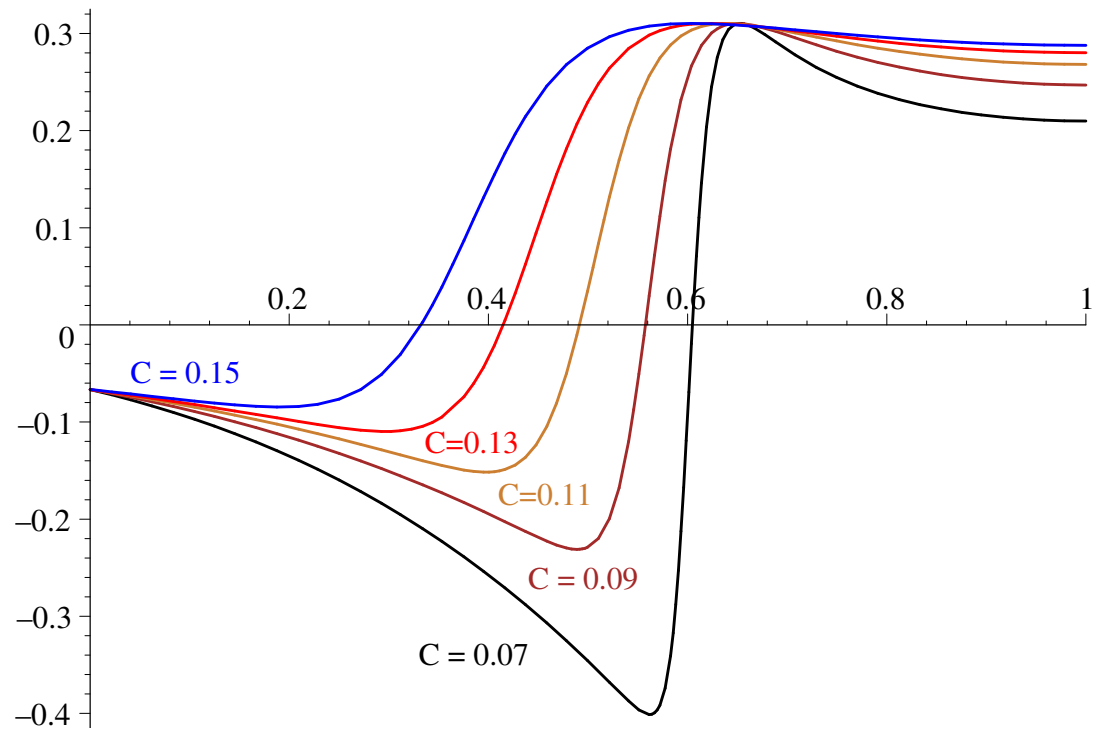

Figure 5: Variation of $\beta(r)$ of Equation (9). 
sign of $\alpha\left(r, r^{\prime}, C\right)$. For the numerical results of Section $3, \alpha\left(r, r^{\prime}, C\right)$ is plotted over all of $[0,1]$ and for a range of (small) $C$-values, as in Figure 4 . Analogous plots for $\beta(r)$ over $[0,1]$ are displayed in Figure 5. The plots of Figures 3, 4 and 5 display several significant features. Figure 3 shows that the radius profile has an inflexion point $z=z_{1}$ (that is, where $r^{\prime \prime}\left(z_{1}\right)=0$ ) near to $z=a$. Figure 4 shows that $\alpha$ has a zero $z=z_{2}$ near to $z=a$ (and $z=z_{1}$ ); and that $\alpha<0$ on $\left[0, z_{2}\right)$. Figure 5 shows that $\beta(r)$ displays a zero at $z=z_{3}$ near all of $a, z_{1}$ and $z_{2}$; and from (11), see that this corresponds to

$$
r=r_{3}=\sqrt{\frac{f_{0}-B}{3 B}} .
$$

In the analysis to follow, we choose $a=z_{2}$; that is, we centre the layer region on the zero of $\alpha$.

While clearly $a$ varies with $C$, the variation is not great; so it is plausible that the solution $r_{1}$ of (9) on $[0, a]$ satisfying the first of (12) is mimicked, in some sense, by that of the semilinear equation

$$
C^{2} r^{\prime \prime}+k(z) r^{\prime}+\beta(r)=0,
$$

where $k(z)$ is a function such that $k(z)<0$ on $[0, a)$ and $k(a)=0$. Recall that $C$ is small, then from standard perturbation theory results [3], such a solution will display a layer of thickness $\mathcal{O}\left(\sqrt{C^{2}}\right)$, that is, $\mathcal{O}(C)$, to the left of $z=a$. In this case, the method of matched expansions constructs an approximation to the solution over the subinterval $[0, a]$.

On $[0, a)$, and bounded away from $z=a$ as $C \rightarrow 0$, we solve Equation (2) using a perturbation expansion in $C^{2}$, subject to the boundary condition at $z=0$. This is our outer expansion [3] on the first subinterval. Note that in this construction, we regard $C^{2}$ as small; but the $C$ term in (2) is retained, so that in this region, $r_{1}$ is determined as the solution of

$$
-6 C r_{1}^{\prime}+4 B r_{1}^{3}-r_{1}\left(f_{0}+B\left(r_{1}^{2}-1\right)\right)=0, \quad r_{1}(0)=1,
$$

which is readily obtained by standard methods. 
In the $\mathcal{O}(C)$ neighbourhood to the left of $z=a$, we expect $r_{1}$ to be given by the solution of an equation of the form of (14) for a suitably determined $k(z)$. To do this, we seek an explicit estimation of $\alpha\left(r, r^{\prime}, C\right)$ in this layer region. Here, $r_{1}^{\prime}$ is large (and positive); and we seek an estimate for this quantity. Recalling that $z=z_{1}$, the point of inflexion of $r$ is near to $z=a$, we set $r^{\prime \prime}=0$ in Equation (2) and solve for $r^{\prime}$ to obtain

$$
r^{\prime}=r_{1,2}^{\prime}=\frac{3 \pm \sqrt{9-\left(3 B r^{2}-f_{0}+B\right)^{2} r^{2}}}{C r\left(3 B r^{2}-f_{0}+B\right)} .
$$

Substitution of $r_{1,2}^{\prime}$ into (10) gives an estimate of $\alpha$ in the layer, as a function of $r$. We also note that $r \approx r_{3}$ there; so that applying (13) to (16) will give values to $r_{1,2}^{\prime}$. Thus, an estimate of $\alpha^{\prime}$ in the layer is

$$
\alpha^{\prime} \approx \frac{\alpha(z=a)-\alpha\left(r=\sqrt{\frac{f_{0}-B}{3 B}}\right)}{C},
$$

which gives, after manipulation,

$$
\alpha^{\prime} \approx \frac{27 B}{4\left(f_{0}-B\right)^{2}}=M, \quad \text { say } .
$$

(Note that one of the values of $r_{1,2}$ gives $\alpha^{\prime} \approx 0$, which we regard as unrealistic.)

So, throughout the layer,

$$
\alpha \approx M(z-a)
$$

so that in the layer to the left of $z=a$, the governing differential equation for $r_{1}$ is

$$
C^{2} r_{1}^{\prime \prime}+M(z-a) r_{1}^{\prime}+\beta\left(r_{1}\right)=0,
$$

where $M$ is as above. The functional form of $\beta\left(r_{1}\right)$ given by (11) makes the differential equation (20) well nigh impossible to solve. However, we argue 
that the essential nature of the solutions of (20) are governed by the first two (derivative) terms; so we set $\beta\left(r_{1}\right)=0$ in (20) and we use the remaining terms to construct the boundary layer function $r_{1}$. Thus, in terms of the local variable $\xi=(a-z) / C$, the differential equation governing an approximate solution in the layer left of $z=a$ is

$$
\frac{d^{2} \tilde{r_{1}}}{d \xi^{2}}+M \xi \frac{d \tilde{r_{1}}}{d \xi}=0,
$$

where $\tilde{r_{1}}(\xi, C) \equiv r_{1}(a-C \xi, C)$, and primes denote derivatives taken with respect to $\xi$. This may be solved subject to the boundary condition $\tilde{r_{1}}(0)=$ $\lambda$. If the results of this calculation, and that for $r_{1}$ above, are combined using the matching process, then a leading order approximation to $r_{1}$ valid over all of $[0, a]$ (that is, the leading term of a composite expansion) is found to be

$$
r_{1 c}(z, C)=\phi(z, C)+(\phi(a, C)-\lambda) \operatorname{erf}\left(\frac{\sqrt{2 M}(a-z)}{2 C}\right)+\lambda-\phi(a, C),
$$

where

$$
\phi(z, C)=\left\{\left(1-\frac{3 B}{f_{0}-B}\right) \exp \left[\left(\frac{f_{0}-B}{3 C}\right) z\right]+\frac{3 B}{f_{0}-B}\right\}^{-1 / 2},
$$

and $M$ is as given above.

On $[a, 1]$, the situation is different. As Figure 4 shows, while $\alpha>0$ there, it is also very small there. Thus, whereas standard theory would indicate a layer to the right of, and adjacent to $z=a$, we anticipate this effect to be small.

We thus seek a simple equation which approximates the full nonlinear equation (2) over all of $[a, 1]$. The profiles of Figure 3 imply that, on this subinterval, $r(z)$ does not vary a lot, while $r^{\prime}(z)$ is bounded. A reasonable 
estimate over $[a, 1]$ is $r(z) \approx r(1)=\rho_{\mathrm{BU}}$. Thus, we propose that on $[a, 1]$ the nonlinear equation (2) be replaced by the approximate equation

$$
C^{2} r_{2}^{\prime \prime}-\frac{3 C r_{2}^{\prime}}{\rho_{\mathrm{BU}}^{2}\left(f_{0}+B\left(\rho_{\mathrm{BU}}^{2}-1\right)\right)}-\frac{\left(f_{0}-B\left(3 \rho_{\mathrm{BU}}^{2}+1\right)\right)}{2 \rho_{\mathrm{BU}}\left(f_{0}+B\left(\rho_{\mathrm{BU}}^{2}-1\right)\right)}=0,
$$

which is readily solved subject to the second of (12), to give a leading order approximation to the radius profile on $[a, 1]$ as

$$
r_{2}(z, C)=\lambda+\frac{1}{18} \rho_{\mathrm{BU}}^{3} \sigma \gamma\left(e^{\eta(z, C)}-e^{\eta(a, C)}\right)-\frac{\rho_{\mathrm{BU}}}{6 C} \gamma(z-a),
$$

where

$$
\begin{array}{ll} 
& \sigma=\left(f_{0}+B\left(\rho_{\mathrm{BU}}^{2}-1\right)\right), \\
& \gamma=\left(f_{0}-B\left(3 \rho_{\mathrm{BU}}^{2}+1\right)\right), \\
\text { and } \quad & \eta(z, C)=\frac{3(z-1)}{C \sigma \rho_{\mathrm{BU}}^{2}} .
\end{array}
$$

The boundary conditions (12) ensure that $r_{1 c}$ and $r_{2}$ join continuously at $z=a$. A smooth join may be obtained (at least to leading order) by imposing the conditions

$$
r_{1 c}^{\prime}(a)=r_{2}^{\prime}(a), \quad r_{2}(1)=\rho_{\mathrm{BU}},
$$

which serve to determine approximate values for $a$ and $\lambda$.

An approximate film radius profile $r_{c}(z)$ valid over all of $[0,1]$ is then

$$
r_{c}(z)=r_{1 c}(z)(H(z)-H(z-a))+r_{2}(z)(H(z-a)-H(z-1)),
$$

where $H(z)$ is the Heaviside function.

\section{$5 \quad$ Results and discussion}

Approximations $r_{1 c}$ and $r_{2}$ were constructed as in the previous section, using the data set

$$
B=0.21, \quad C=0.15, \quad f_{0}=0.969, \quad \rho_{\mathrm{BU}}=3.85 .
$$




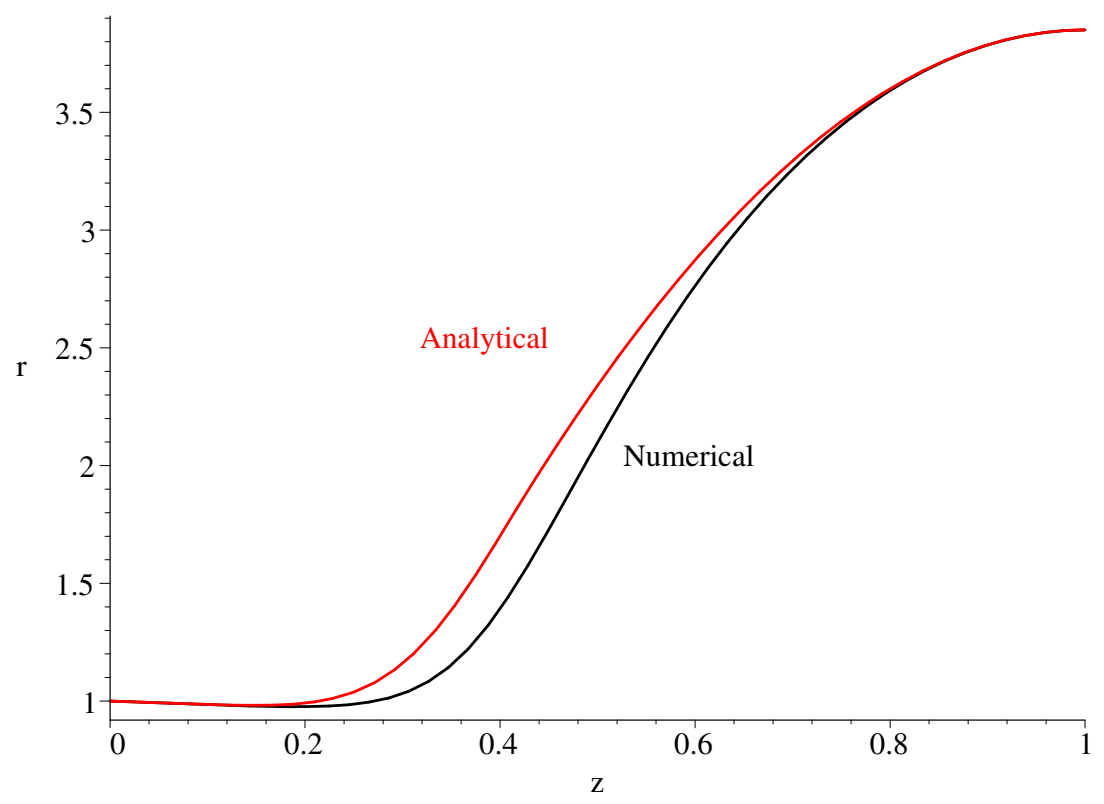

Figure 6: Comparison of bubble radius profiles as given by (30) and numerical calculations. The dotted curve is the numerical result.

The conditions (29) gave

$$
a \approx 0.4100437 \text { and } \lambda \approx 1.7692546,
$$

and the overall approximation $r_{c}(z)$ constructed by (30). Figure 6 compares the results of numerical calculations using the package Maple with the results obtained above. Agreement between the two profiles seems quite good, given the somewhat rough estimates used in obtaining the approximating differential equations (20) and (24). As anticipated, the discrepancy is greatest in the layer region. Nevertheless, both profiles have much the same structure, with the rapid transition centred about more or less the same point in each case. The analysis used here was motivated by a desire to obtain a straightforward analytical expression for the radial film bubble profile, that minimized calculation, and provided reasonable accuracy. We feel that the above calculations have largely achieved this. 


\section{References}

[1] J. C. Bennett, Interior Layer Structure in the Blown Newtonian Film, Honours Thesis, School of Mathematical and Geospatial Science, RMIT, 2004. C845

[2] X.-L. Luo, R. I. Tanner, A Computer Study of Film Blowing, Polymer Engineering and Science, Vol.25, no.10, 1985, pp.620-629. C845

[3] A. H. Nayfeh, Perturbation Methods, John Wiley and Sons, 1973. $\mathrm{C} 848$

[4] J. R. A. Pearson, Mechanics of Polymer Processing, Elsevier Applied Science Publishers, 1985. C842

[5] J. J. Shepherd, H. J. Connell and D. C. H. Tam, An interior layer in a film-blowing problem, Proceedings of the Fifth Biennial Engineering Mathematics and Applications Conference (EMAC2002),

M. Pemberton, I. Turner, P. Jacobs (eds), The Institution of Engineers Australia, Brisbane, Queensland (2002), pp.181-186 C845

[6] D. C. H. Tam, Mathematical Analysis of the Blown Newtonian Film, MAppSc Thesis, RMIT, 2003 C845

[7] D. C. H. Tam, J. Shepherd and H. Connell, Modelling the film blowing process, Proceedings of the Fourth Biennial Engineering Mathematics and Applications Conference (EMAC2000), R. L. May, G. F. Fitz-Gerald, I. H. Grundy (eds), The Institution of Engineers Australia, Melbourne (2000), pp.271-274. C845 